\title{
Kinetics matter: prognostic implications of early bone marrow assessment in acute myeloid leukemia
}

Florian Perner ${ }^{1,2}$

${ }^{1}$ Department of Pediatric Oncology, Dana Farber Cancer Institute, Harvard Medical School, Boston, MA, USA and ${ }^{2}$ Innere Medizin C, Universitätsmedizin Greifswald, Greifswald, Germany

E-mail: Florian_Perner@DFCl.harvard.edu

https://doi.org/10.3324/haematol.2021.280133

(อ2022 Ferrata Storti Foundation

Haematologica material is published under a CC-BY-NC license @( ) \&

Despite recent advances in molecular stratification and targeted therapies, including the availability of BCL2-, FLT3- and IDH1/2-inhibitors, curative treatment of patients with acute myeloid leukemia (AML) remains dependent on intensive treatment regimens based on high-dose chemotherapy and consolidation with allogenic hematopoietic stem cell transplantation (HSCT). In order to ensure optimal outcomes for patients it is essential to balance the intensity of the therapeutic interventions and properly target the malignant cells but limit toxicity if possible. A well-defined risk stratification system by the European LeukemiaNet (ELN) classifies patients into "favorable", "intermediate" or "adverse" risk groups based on predictive genetic determinants and provides riskadjusted therapeutic recommendations. This classification system has proven to be effective in clinical routine and is internationally accepted. Nevertheless, the currently established therapeutic decision trees are fairly static, incorporating mainly genetic factors and the evaluation of response at the end of each treatment cycle. Several studies have proposed, that the kinetics of remission induction may be an independent prognostic factor to guide the decision of whether treatment should be intensified to improve long-term outcomes. ${ }^{2-4}$ Due to

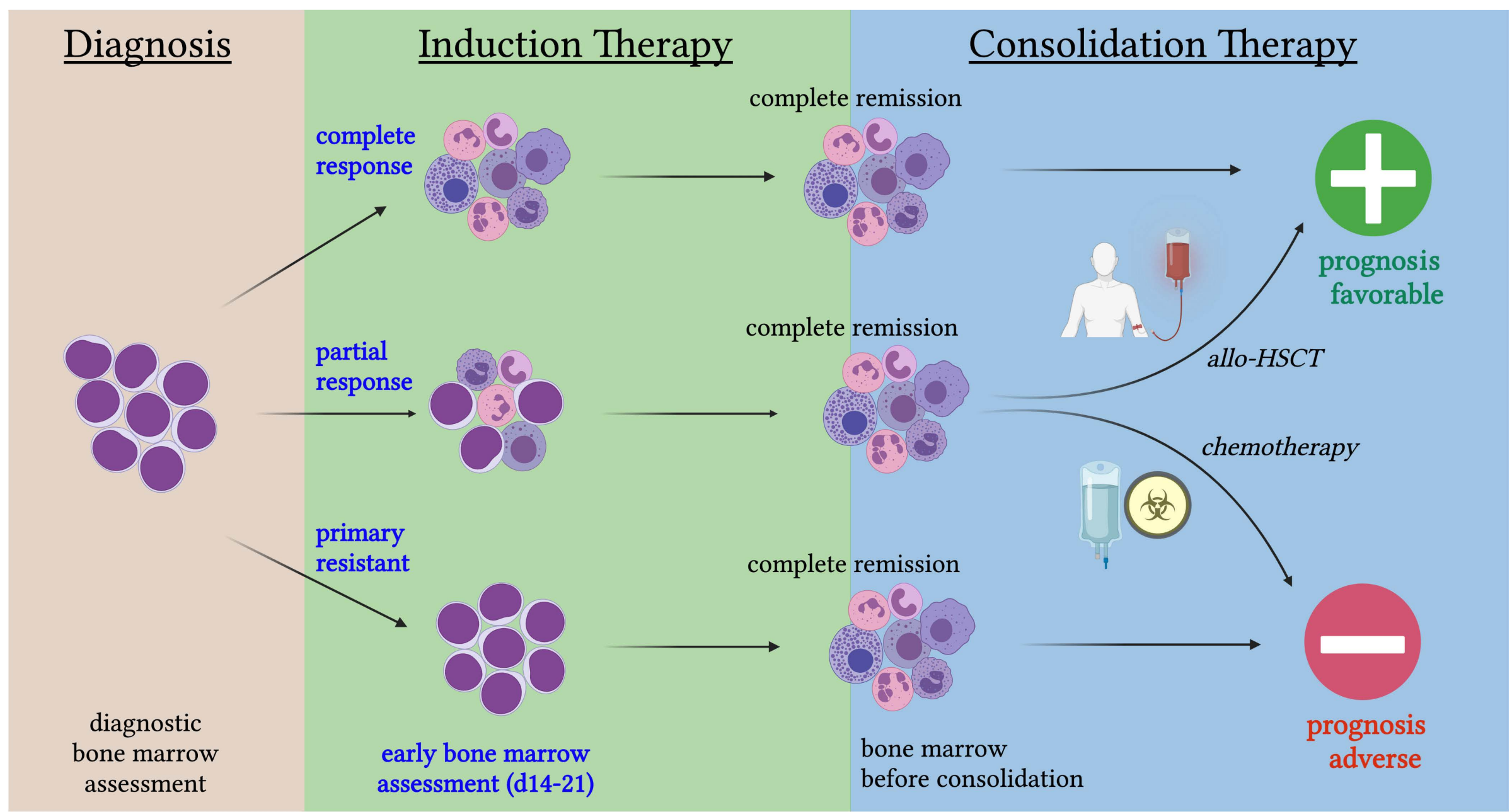

Figure 1. Prognostic relevance of early bone marrow assessment in acute myeloid leukemia. Schematic depicting the key findings of Ihlow et al. regarding the prognostic relevance of early bone marrow assessment within the population of patients with acute myeloid leukemia, who reach complete remission after intensive induction chemotherapy. A complete clearance of blasts between day 14 and 21 is a favorable prognostic factor, while early resistant disease is an adverse factor even when a complete remission is reached before consolidation. Importantly, the negative prognostic impact of a partial response at early bone marrow assessment may be overcome if patients are consolidated with allogenic stem-cell transplantation but not conventional chemotherapy. allo-HSCT: allogeneic hematopoietic stem cell transplantation. 
the relatively small numbers of patients examined in most of these studies and/or the lack of long-term follow-up data no clear consensus has yet been established in the field on whether the kinetics of blast clearance should be taken into consideration for therapeutic decision-making.

In this issue of Haematologica, Ihlow et al. report on their findings from a retrospective study in 1,008 patients undergoing intensive treatment for AML. ${ }^{5}$ The authors aimed to clarify the question whether early bone marrow assessment (day 14-21) during the first cycle of induction chemotherapy has a predictive value for long-term clinical outcomes, specifically within the majority of patients who reach a complete remission before consolidation (Figure 1). Due to high patient numbers and long-term follow-up, Ihlow and co-authors were able to perform sophisticated analyses of various subgroups of patients and add significant granularity to the concept of early response assessment in AML.

Not surprisingly, early blast clearance was a favorable prognostic factor both in patients who underwent consolidation by chemotherapy and those who underwent allogenic HSCT. Similarly expected, patients who failed to clear leukemia cells during induction therapy had inferior outcomes compared to patients who reached complete remission across all subgroups. Interestingly, Ihlow et al. discovered relevant prognostic ramifications within the subgroup of patients who showed blast persistence at the point of early bone marrow assessment (day 14-21) but eventually reached complete remission before consolidation. The authors were able to demonstrate, that patients with "early resistant" disease had significantly worse outcomes after both allogeneic HSCT and chemotherapy consolidation, even when reaching complete remission after the last cycle of induction therapy. Of note, patients with blast persistence reaching the status of a partial remission during early bone marrow assessment followed by a complete remission after induction did not have a significant survival disadvantage over patients with early blast clearance when consolidated with allogeneic HSCT. In contrast, an early partial remission followed by complete remission after induction was associated with a significantly decreased progressionfree survival when patients were consolidated with chemotherapy (Figure 1).

Implications of the work presented by Ihlow et al. will be particularly relevant as instrument for improved decision making in the subset of patients diagnosed with ELN "intermediate"-risk AML, for whom the recommendations for allogeneic HSCT are not strictly defined. According to the authors, the application of a decision-making concept incorporating the findings from their current study would have changed therapeutic decisions in about a third of patients with "intermediate"-risk AML. Furthermore, one could speculate that even patients with "favorable"-risk AML may benefit from allogeneic HSCT in case of early blast persistence. Going forward, the utility of these exciting concepts must be confirmed in prospective clinical trials to allow a precise assessment of which groups of patients will benefit from allogeneic HSCT based on early blast persistence.

The fact that allogeneic HSCT but not consolidation with conventional chemotherapy was able to overcome the negative prognostic impact of an early partial remission implies that early response assessment primarily reflects cell-intrinsic differences in molecular networks affecting sensitivity to cytotoxic agents. It is tempting to speculate which genetic and/or epigenetic factors may be drivers of this increased resistance to chemotherapy and how far molecular profiling may be useful in the future to further stratify these patients and objectify therapeutic decisions based on genetic markers.

\section{Disclosures}

No conflicts of interest to disclose.

\section{References}

1. Dohner H, Estey E, Grimwade D, et al. Diagnosis and management of AML in adults: 2017 ELN recommendations from an international expert panel. Blood. 2017;129(4):424-447.

2. Kern W, Haferlach T, Schoch C, et al. Early blast clearance by remission induction therapy is a major independent prognostic factor for both achievement of complete remission and longterm outcome in acute myeloid leukemia: data from the German AML Cooperative Group (AMLCG) 1992 Trial. Blood. 2003;101(1):64-70.

3. Hussein K, Jahagirdar B, Gupta P, Burns L, Larsen K, Weisdorf D.
Day 14 bone marrow biopsy in predicting complete remission and survival in acute myeloid leukemia. Am J Hematol. 2008;83(6):446-450.

4. Estey EH, Shen Y, Thall PF. Effect of time to complete remission on subsequent survival and disease-free survival time in $A M L$, RAEB-t, and RAEB. Blood. 2000;95(1):72-77.

5. Ihlow J, Gross S, Busack L, et al. Acute myeloid leukemia: negative prognostic impact of early blast persistence can be in part overcome by a later remission prior to post-induction therapy. Haematologica. 2022;107(8):1773-1785. 